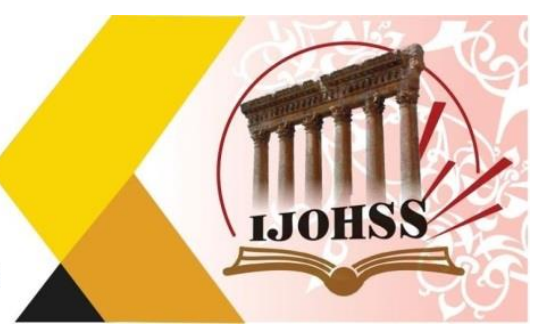

\title{
اثر الحقيبة التعليمية الاكترونية في تحصيل العلوم وحب الاستطلاع العلمي لطلاب الصف الثاني المثوسط الأيز
}

\author{
م مده. احمد حمزة عبود \\ العلوم التربوية والنفسية ــ المناهج وطرة عبود ائق التدريس

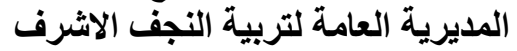

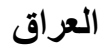

\author{
م.ذه. مسلم محمد جاسم \\ العلوم التربوية والنفسية ـ المناهج وطر جائق مائق التدريس \\ كلية التربية للبنات ـ جامعة القادسية الترجية \\ العراق \\ musjasim@gmail.com \\ muslim.m.jasim@qu.edu.iq
}

الملخص

هدف البحث الى التحقق من اثر الحقيبة التعليمية الالكترونية على تحصيل العلوم وحب الاستطلاع العلمي لطـلاب الصف الثاني المتوسط ، تكون مجتمع البحث من طـلاب الثاني المتوسط في المدارس المتوسطة في محافظة الديو انية ، اختار الباحثنان منها عشو ائيا متوسطة الاسر اء المتكونة من شعبتين للثناني المتوسط اختار عشو ائيا شعبة (أ) كمجمو عة تجريبية مكونة من (44) طالبا ، وشعبة ( ب ) كمجمو عـة ضـابطة مكونـة مـن (42) طالبا فيكون مجموع عينـة البحث (86) طالبـا ، و استخدم الباحثنان التصميم التجريبي ذا المجمو عتين التجريبية و الضـابطة واعد الباحثان أداتين هما اختبار التحصيل للعلوم ومقياس حب الاستطلاع العلمي وبعد التحقق من صدقهما وثباتهمـا تم تطبيقهمـا على عينـة البحث ، استخدم SPSS لحسـاب (الصسوبة ، التمييز، فعاليـة البدائل الخاطئة ، الفا كرونباخ ، t-test لعينتين مستقلتين)، اظهرت نتائج البحث تفوق طـلاب المجموعة التجريبية على طلاب المجمو عة الضابطة في التحصيل وحب الاستطلاع العلمي ، وفي ضوء ذللك توصل الباحثان الى عدد من الاستنتاجات و التوصيات ومنها استخدام الحقيبة التعليمية الالكثرونية في تدريس العلوم ، و اقترحا إجراء در اسـات

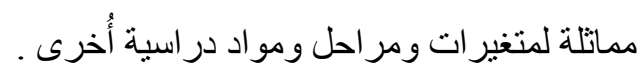

الكلمات المفتاحية: الحقيبة التعليمية الاكترونية، تحصيل العلوم، الصف الثاني المتوسط. 


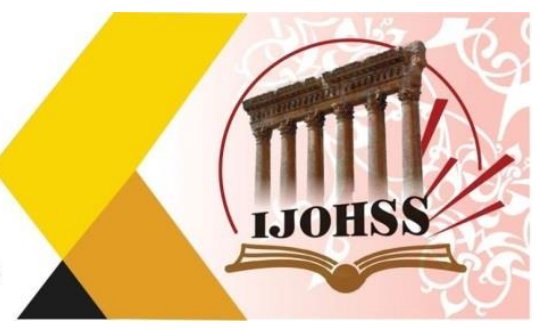

\title{
Effect of Electronic Educational Package on Science Achievement And Scientific Curiosity for Second-Grade Intermediate Students
}

\author{
Dr . Muslim Mohammed Jasim \\ Educational and Psychological Sciences - Curricula and Teaching Methods \\ College of Education for Women - University of Al-Qadisiyah - Iraq \\ musjasim@gmail.com \\ muslim.m.jasim@qu.edu.iq \\ Dr. Ahmed Hamzah Abbood \\ Educational and Psychological Sciences - Curricula and Teaching Methods \\ General Directorate of Education in Al-Najaf - Iraq
}

\begin{abstract}
This research aimed to verify the effect of the electronic educational package on the achievement of science and the scientific Curiosity of students of the second intermediate grade. The research community consisted of students of the second intermediate schools in the province of Diwaniyah. The two researchers randomly selected Al Israa intermediate school, which consisted of two classes of the second intermediate class. (A) class was the experimental group consisting of (42) students, and class (B) as a control group consisting of (44) students, so the total research sample would be (86) students, the researchers used the experimental design with the two experimental and control groups. The two researchers prepared two tools, the science achievement test and the scientific curiosity scale after verifying their validity and reliability, they were applied on the research sample, SPSS was used to calculate (difficulty, discrimination, effectiveness of false alternatives, Cronbach's alpha, t-test for two independent samples), the results of the research showed that the experimental group students outperformed the control group students in achievement and scientific curiosity. In light of this, the two researchers reached a number of conclusions and recommendations, including the use of the electronic educational package in teaching science, and suggested conducting studies similar to variables and stages and other study materials.
\end{abstract}

Keywords: e-learning bag, science achievement, second intermediate grade. 


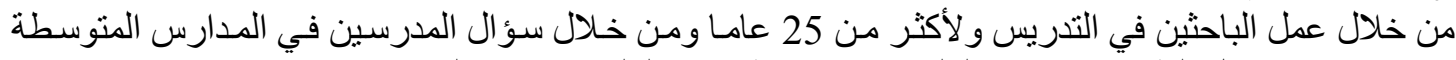

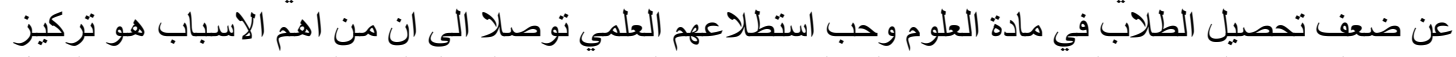

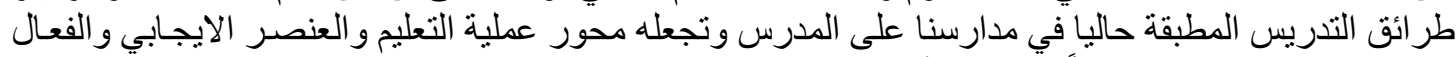

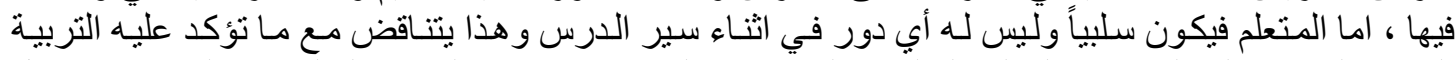

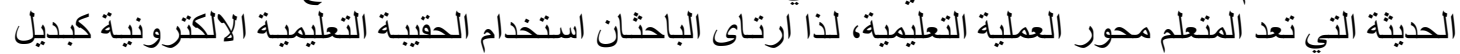

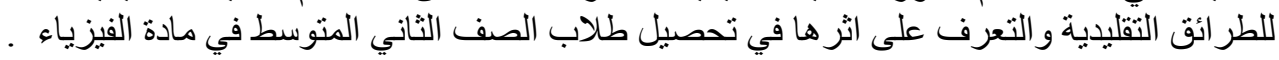

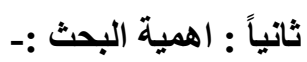

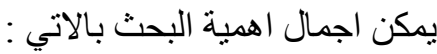

1. مر اعاة الفروق الفردية بين المتعلمين الفين من خلال استخدام الحقيبة التعليمية.

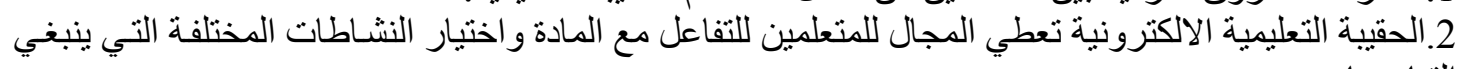

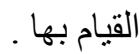
3.ضرورة إتباع أساليب حديثة في تدريس الفيزياء تجعل المتعلم محور اً للعملية التعليمية .

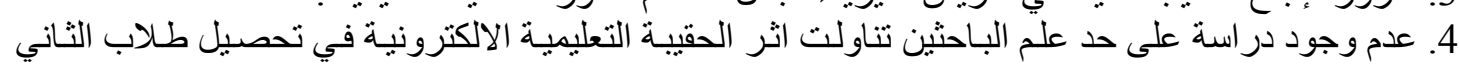
المتوسط في مادة الفيزياء.

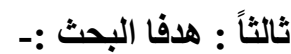
يهدف البحث الحالي إلى معرفة اثلى البر التدريس بالحقيبة التعليمية الالكترونية في :

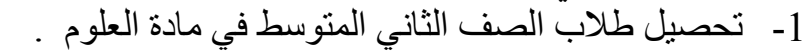
2- ب الاستطلاع العلمي لدى طلاب الصف الصن الثاني المتوسط.

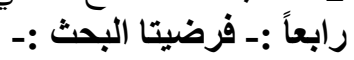

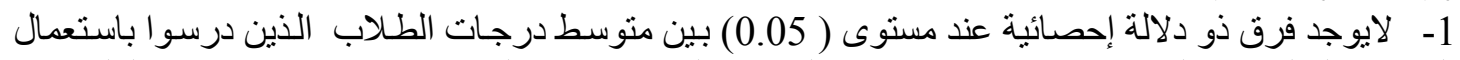

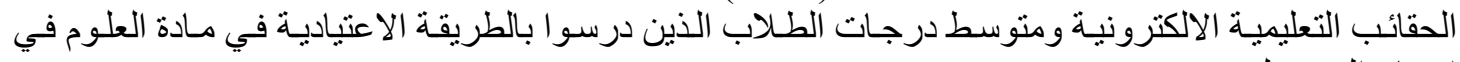

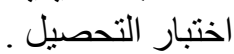

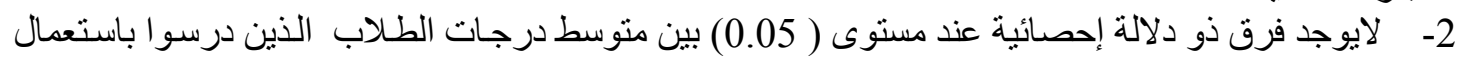

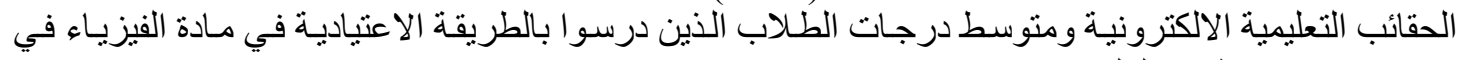
اختبار حب الاستطلاع العلمي . خامساً : حدود البحث :- : بإن

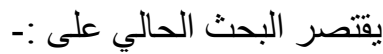
1. ـ طلاب ( الصفّ الثناني المتوسط ) في المدارس المتوسطة في مدينـة الديوانيـة للعام الدراسي (2017 2018 ) الفصل الدراسي الثناني.

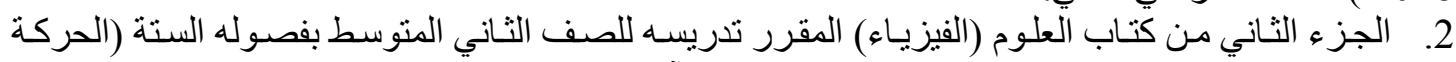

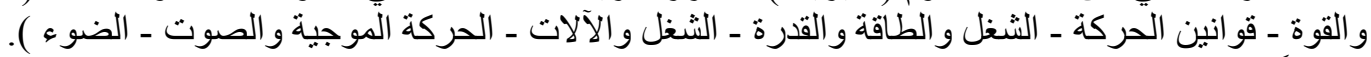

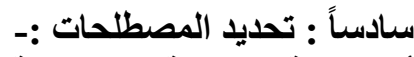
أـ الحقيبة التعليمية الاكترونية :

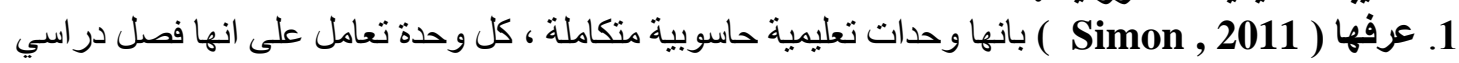

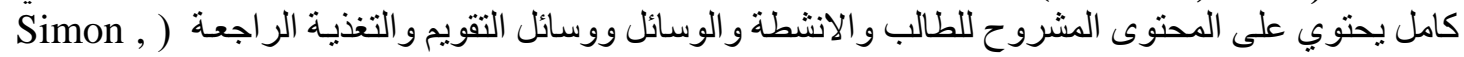




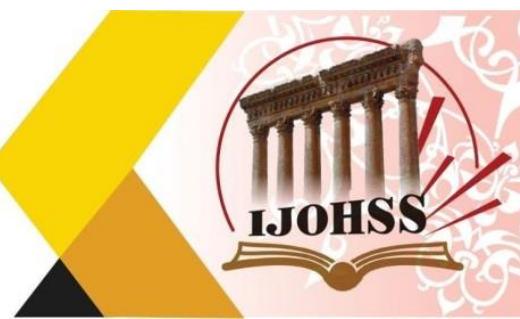

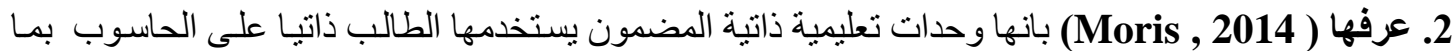

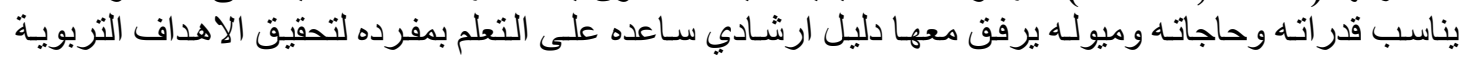
المطلوبة. (Moris , 2014 , 65)

حب الاسنطلاع العلمي

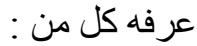
1. (رضوان ، 2012) بأنه دافع لاى المتعلم للتعرف على المو اقف العلمية في البيئة ومحاولة فهمها وتفسيرها

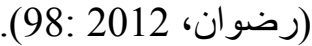
2. (الثمري ، 2016) بحث المتعلم عن اجابات لتساؤلاته المتعلقة بالظواهر العلمبة في بيئته ) (الثمري،

\section{الفقلة الثاني التعليمية الاككترونية}

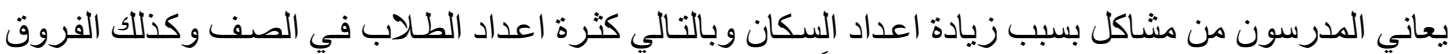

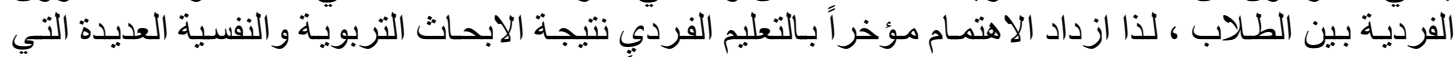

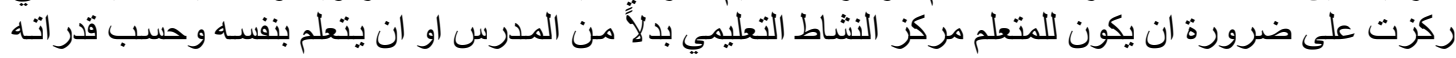

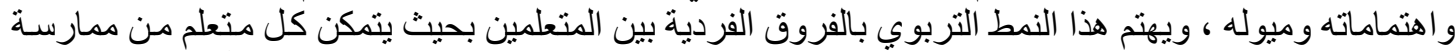

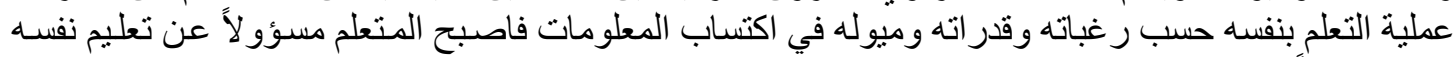

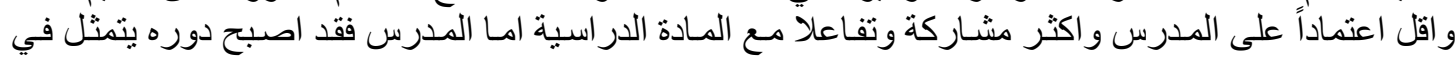

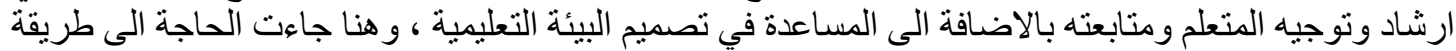

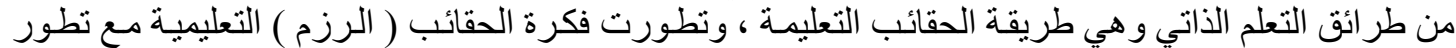

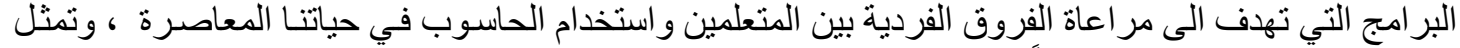

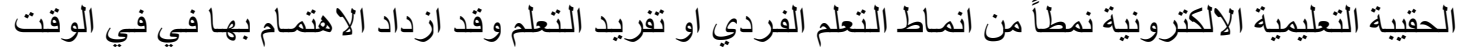

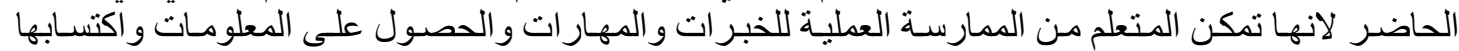

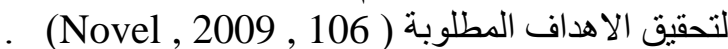

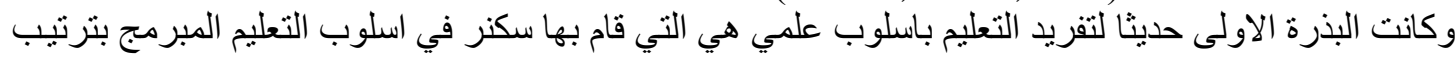

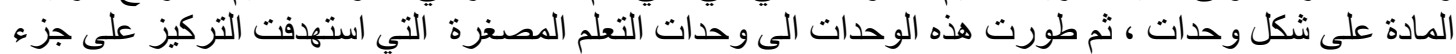

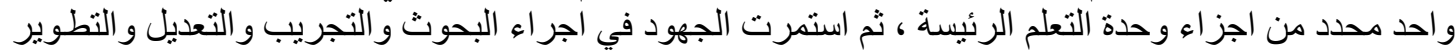

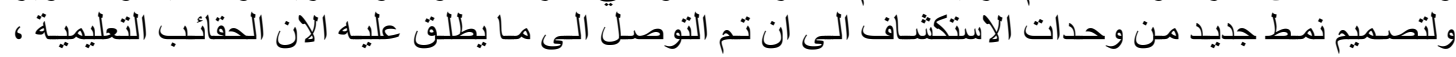

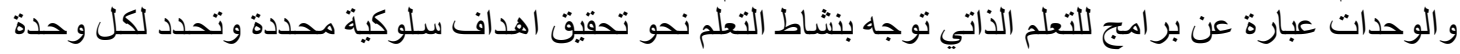

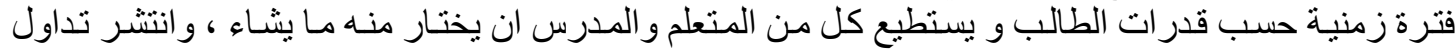

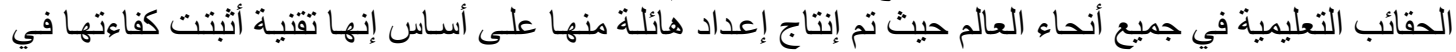

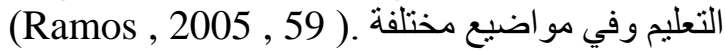

انواع الحقيبة التعليمية الاكترونية :-

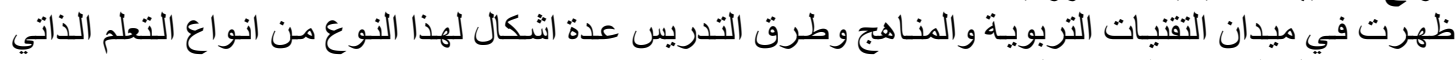
ويتوقف شكل الحقيبة على عو امل منها :-

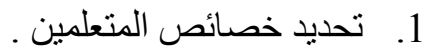
2. 3. الفئة المستهدفة .

ونتيجة للتطور الحاصل في تصميم الحقائب التعليمية ونظر اً للمرونة الكبيرة التي تتمتع بها فقد ظهرت عدة انواع منها :- 
وتعتمد على مو ادو ومصنادر تعليمية جاهزة بدلا من تصميمها ، ويكتفي بفحص تلك الكي المو اد وتحديد المناسب منها

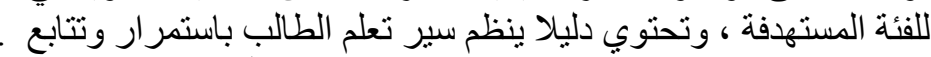

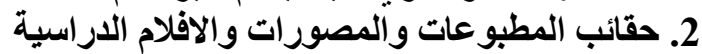

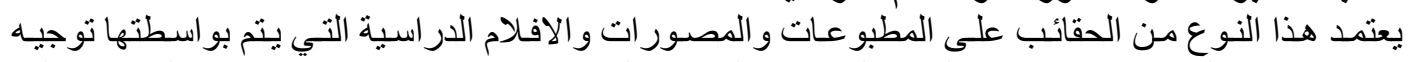

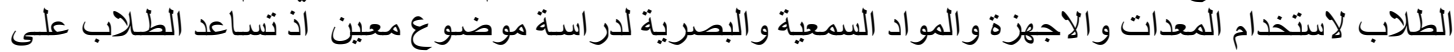

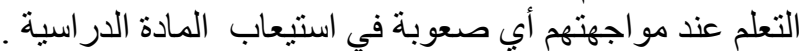
3. حقائب نظام التوجه السمعي التي

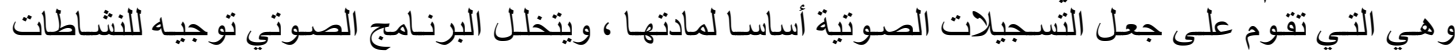

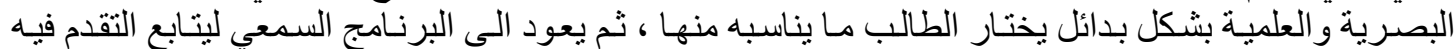

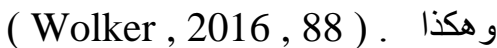

$$
\text { 4. الحقائب المركزة }
$$

5. 5قائب النشاط التعليمية

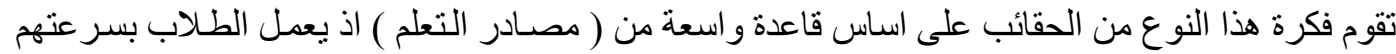

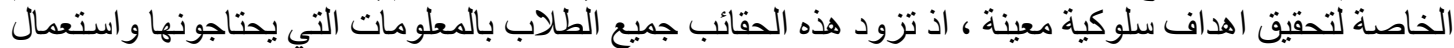

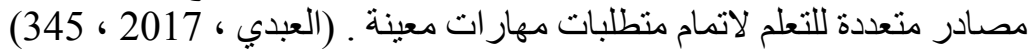

6- الحقائب بالوسائط المتعددة. ( Multi - Media packages)

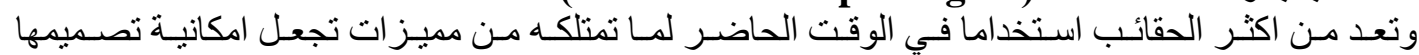

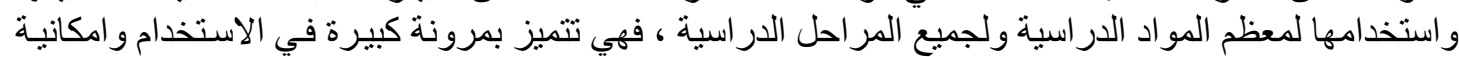

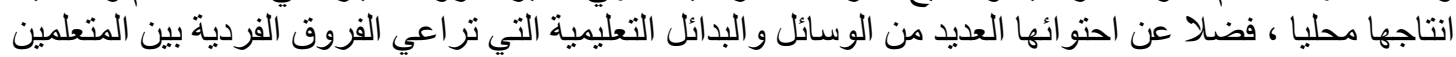

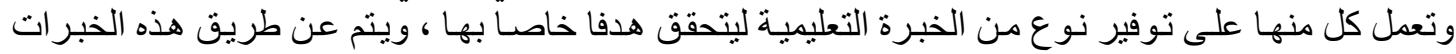

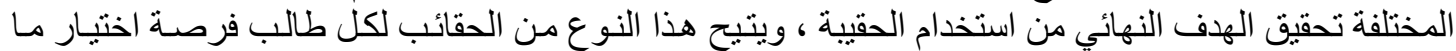

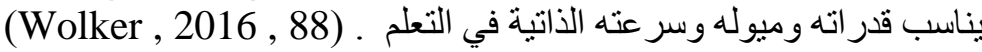
من الوظائف الرئيسة في التعليم هي تنمية حب الاستطلاع و استثارته لتحقيق تعلم و ابداع لدى الطلبة من خلال

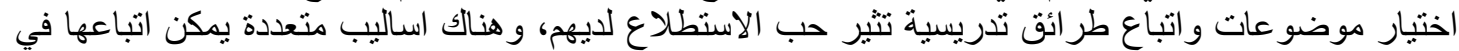

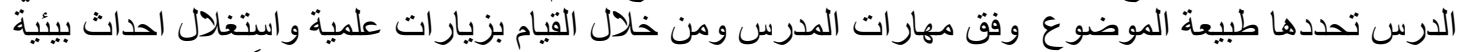

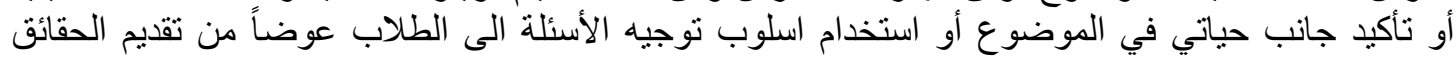

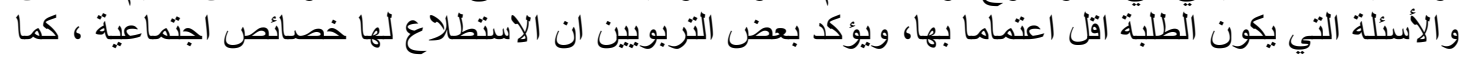

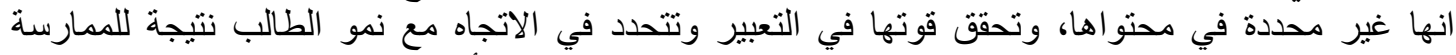

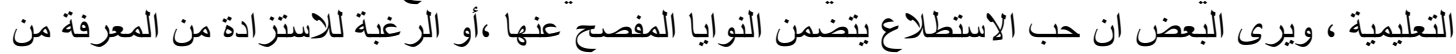

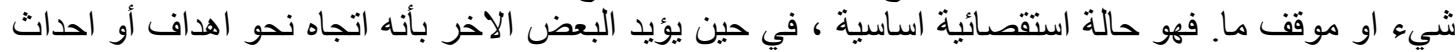

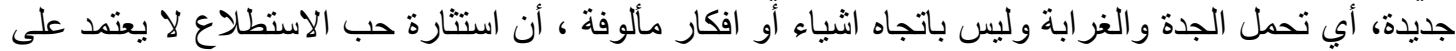

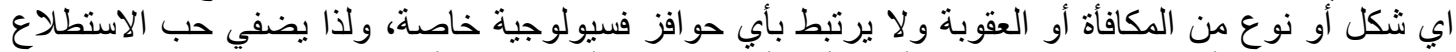

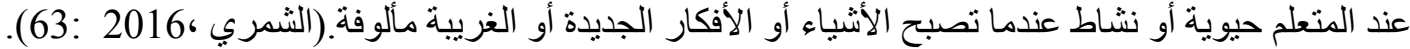

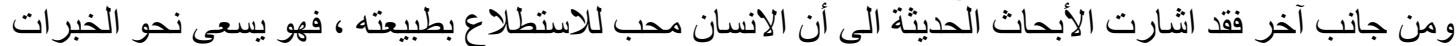

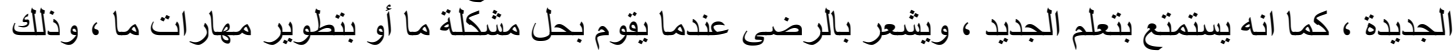

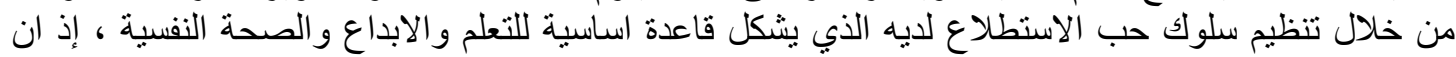




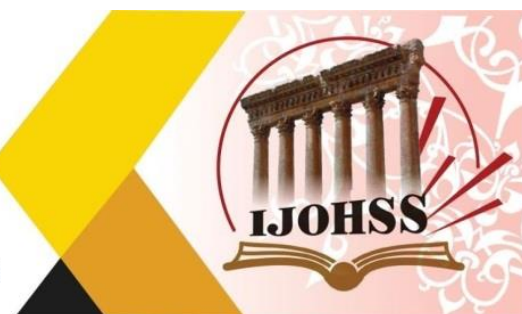

احدى المهمات الرئيسة في التعلم هي كيفية رعاية حب الاستطلاع ، واستغلاله لتحقيق التعلم (رضوان، . (151: 2012

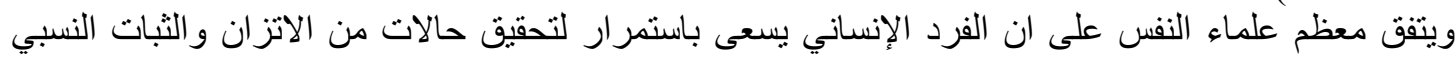

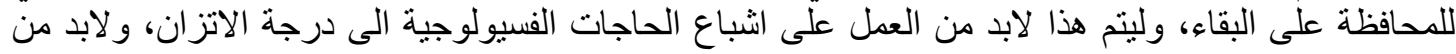

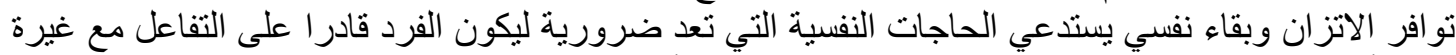

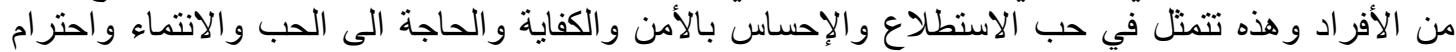

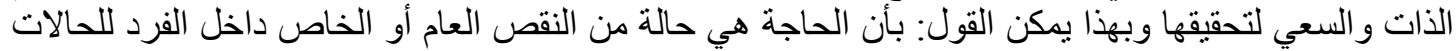

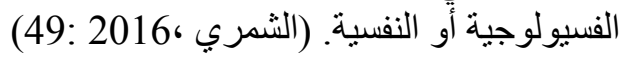

الفصل الثالث

اولاً : منهج البحث والته التصميم التجريبي :

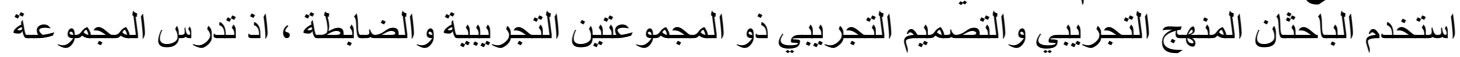

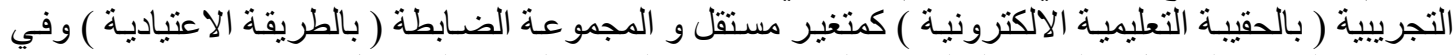
يقاس اثر المتغير المستقل ( الحقيبة التعليمية الالكترونية ) في المتغير التابع ( التحصيل ) .

ثنانياً : مجتمع البحث وعينته.

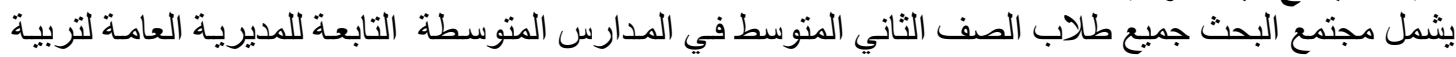

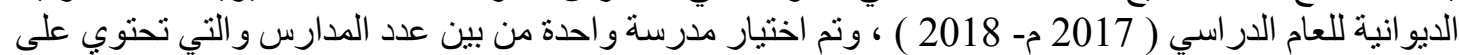

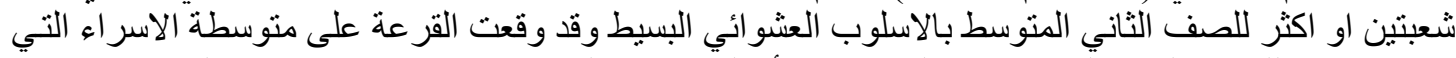

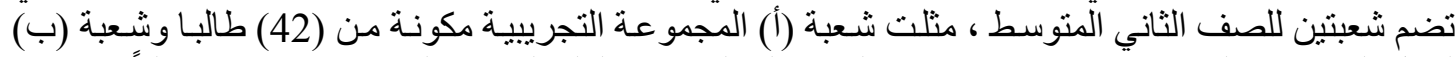
لتمثل المجموعة الضابطة مكونة من (44) طالبا ، وبلغ المجموع الكوع الكلي لطلاب المجمو عتين (86) طالباً

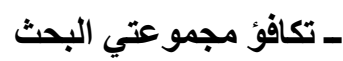

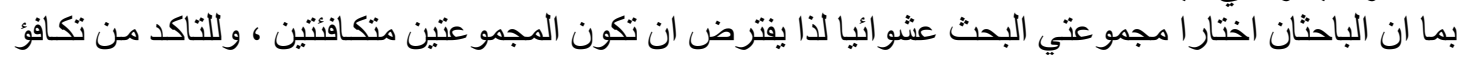

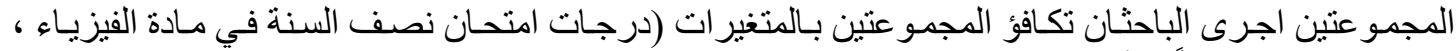

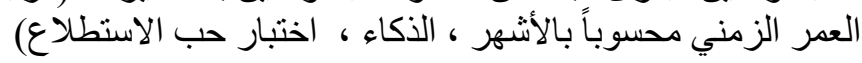

ـ ضبط المتغيرات الاخيلة ( غير التجريبية ) 1. العمليات المتعلقة بالنضج 2.

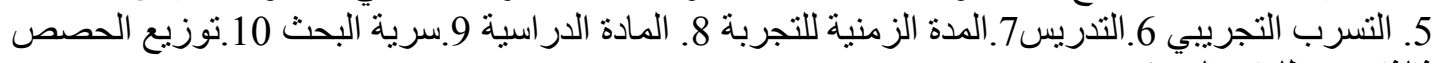

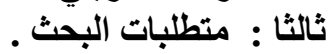

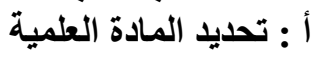

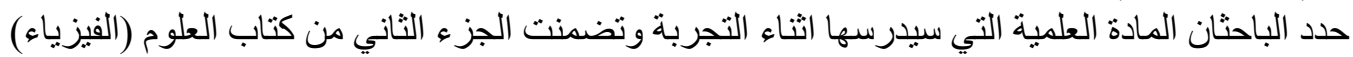
المقرر تدريسه للصف الثاني المنوسط بفصوله الستة (الحركة والقوة ـ قو انين الحركة ـ ـ الثنغل و الطاقة و القدرة -

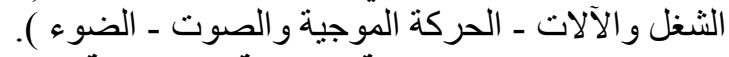

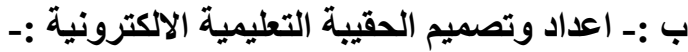

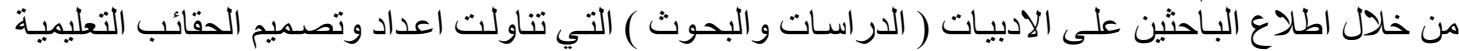

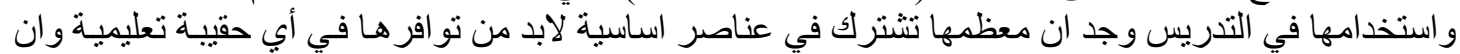

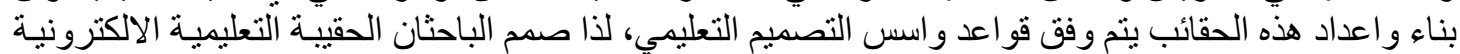

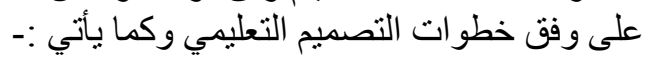

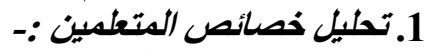

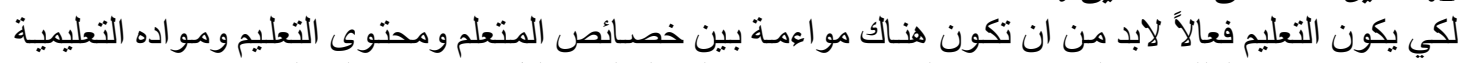

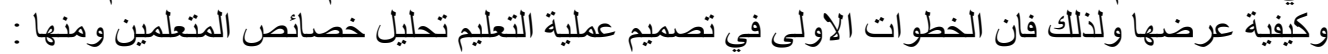


العدد (15) لسبتمبر 2020 Volume (15)
September 2020

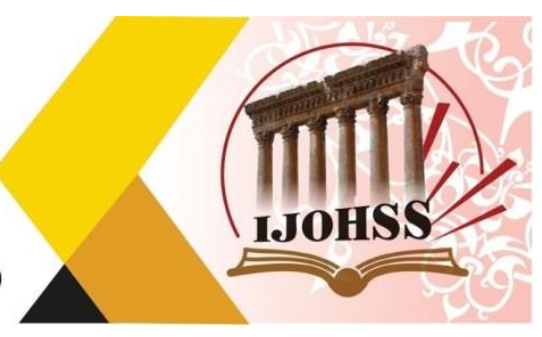

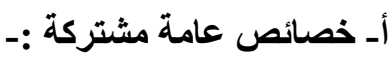

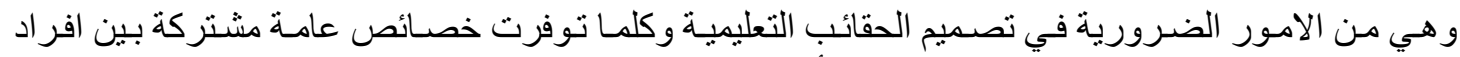

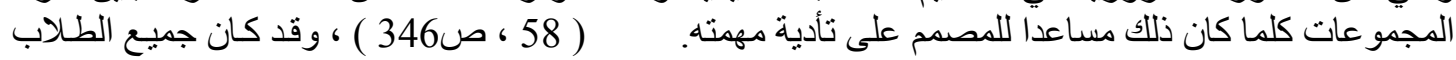

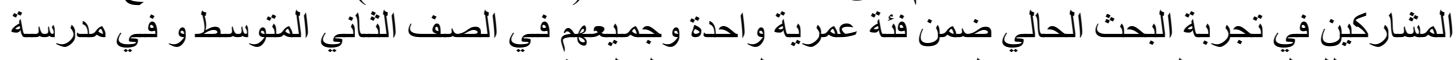

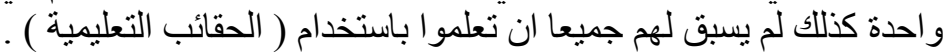

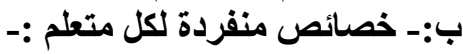

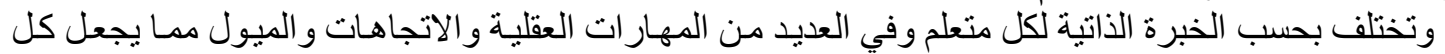

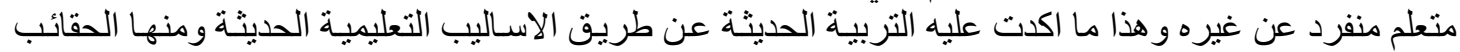

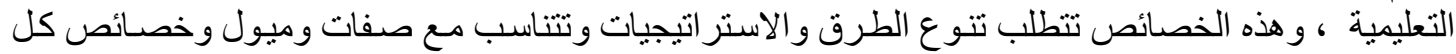

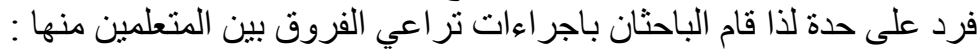

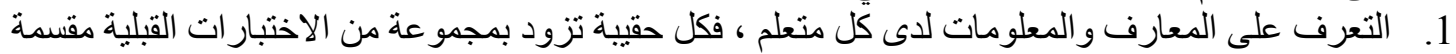

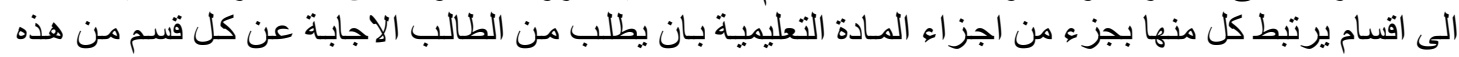

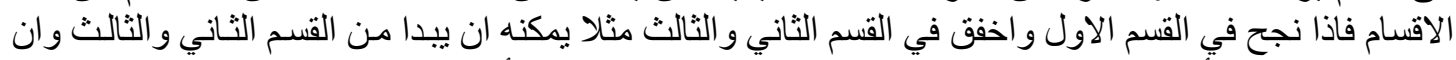

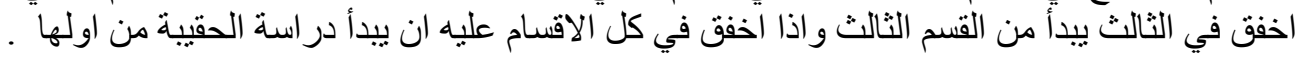

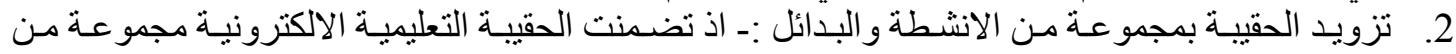

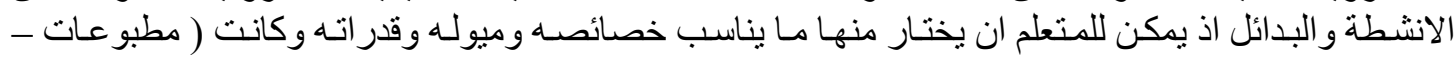

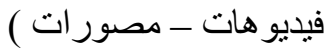
3. ت تحديد جدول زمنسي لدر اسـة الحقائب التعليميـة في ضـو هـ مـا يستغرقه كل فصل (وحدة ) للصف الثاني المتوسط.

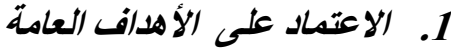

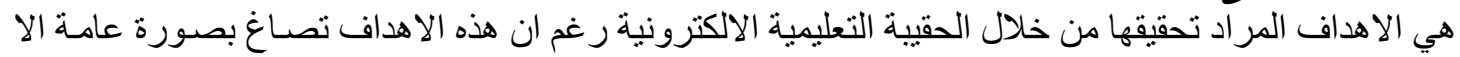

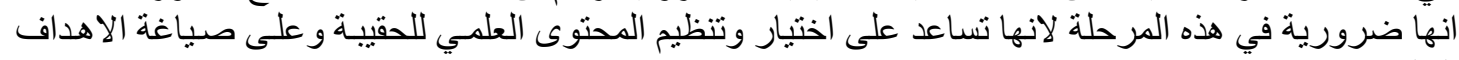

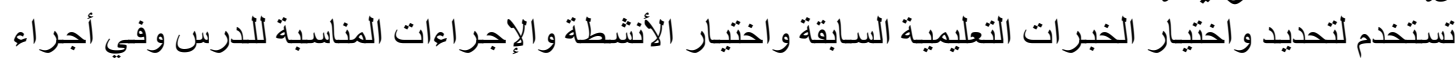

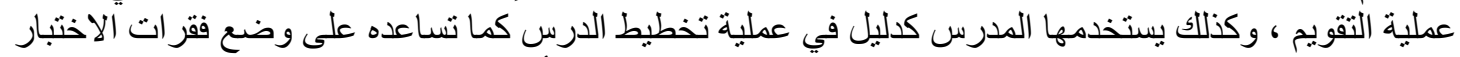

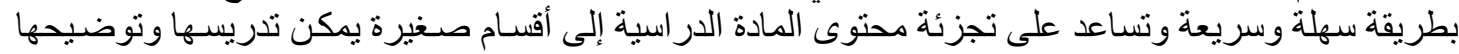

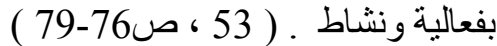
اعد الباحثنان ( 138) هدفا سلوكيا ، وفق المستويات الثلاثة الاولى من تصنيف ( بلوم ) المعرفي ( التذكر - الفهم

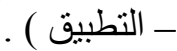

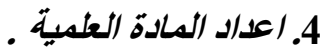

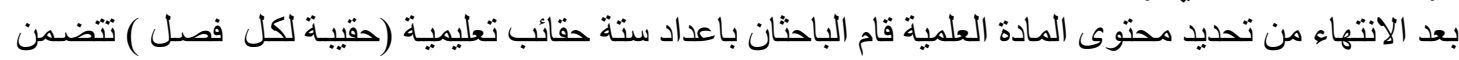

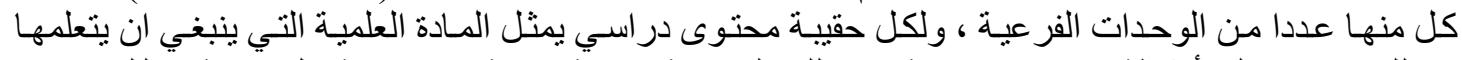

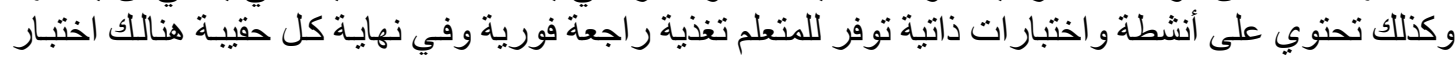

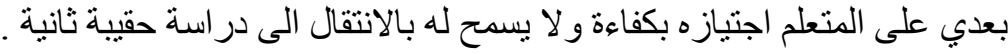

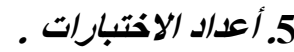

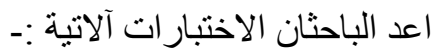

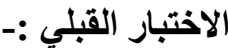

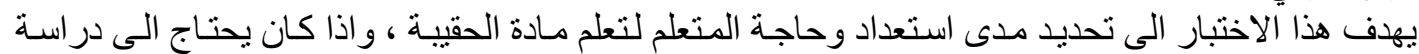

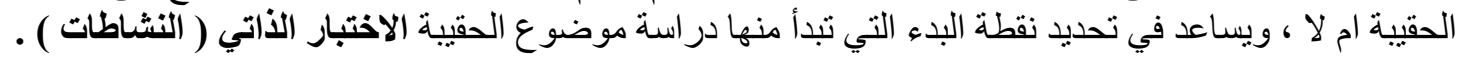




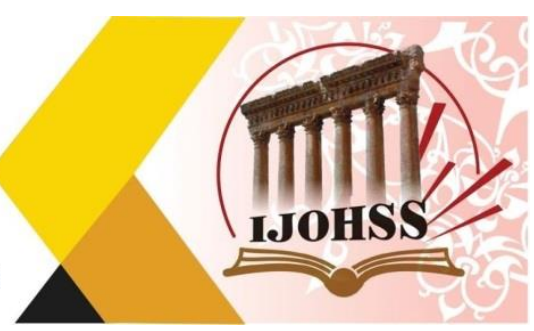

ويعد وسيلة للتغذية الر اجعة الفورية بان يتعرف المتعلم على نقاط الضعف و القوة لديه وجو انب الحقيبة التي ما

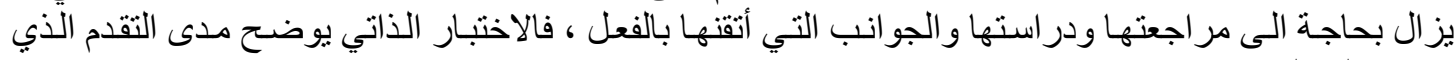

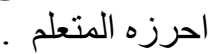

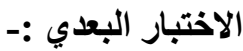

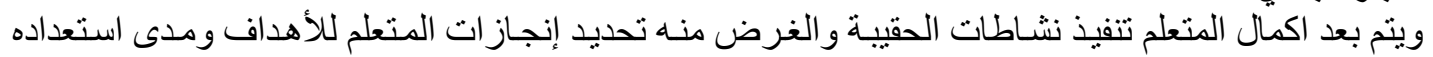
للبدء بالحقيبة التالية بتحديد درجة النجاح و التمكن من الوحدة الدر اسية. 6 أعداد الوحدات التعليمية.

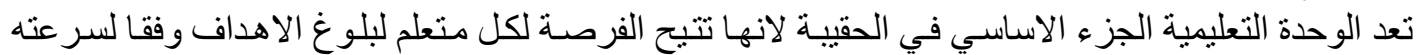

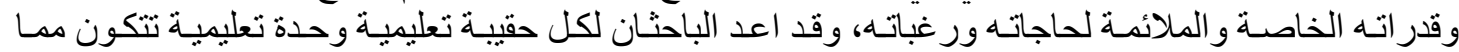
1. باجي :-جهة الحقيبة التعليمية الاككترونية :- ويشتمل على ( عنوان الحقيبة ،، عنوان الوحدة الدراسية والصف الدر اسي ) . 2. عنوان الحقيبة : هو اول مكونات الحقيبة التعليمية الاكترونية والذي يوضح الفكرة الاساسية التي تعالجها

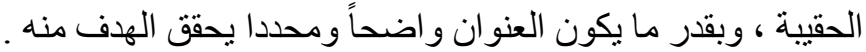

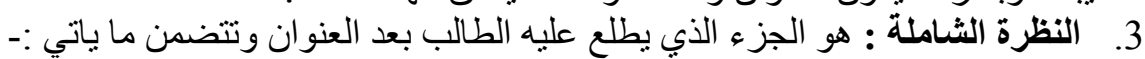
ـالفئة المستهدفة : و هم طلاب الصف ألماء الثناني المتوسط.

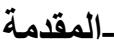
ـالإرشـادات والتعليمـات :- تتضمن كل حقيبة تعليميـة مجموعة من الارشـادات و التعليمـات التي توضـح للمتعلم

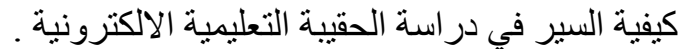
الأهداف السلوكية تحديد الأهداف المر اد تحقيقها في نهاية كل حقيبة تعلية تليمية ، ويتم عرضهها قبل بدأ الدراسـة في الحقيبة التعليمية.

4. المادة العلمية : اعدت بشكل اجز اء متسلسلة مشوقة وفقا لحاجات المتعلمين و الأهداف التي تمت صياغتها.

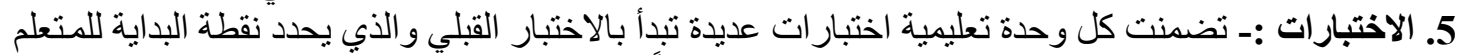

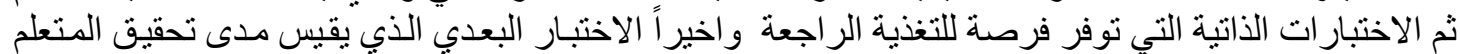
كالافداف الحقيبة التعليمية الالكترونية.

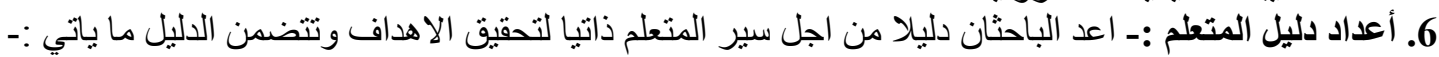

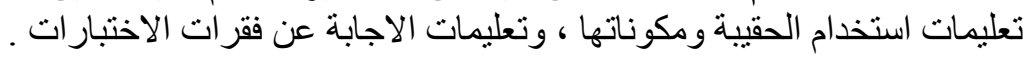

\section{صلاحية الحقيبة التعليمية الاكترونية}

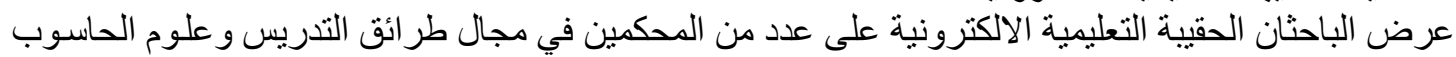

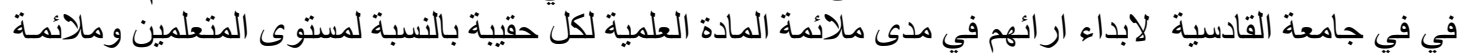

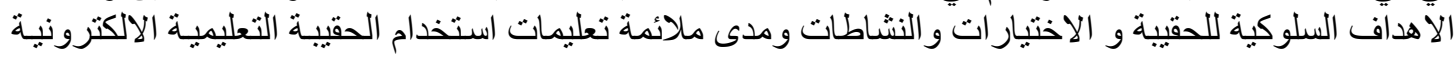

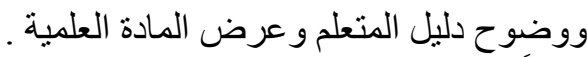

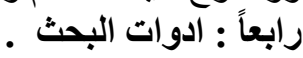

يتطلب البحث الحالي اعداد اختبارين لقياس تحصيل العلوم وحب الاستطلاع العلمي للمجمو عتين ( التجريبية

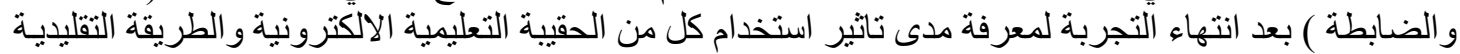

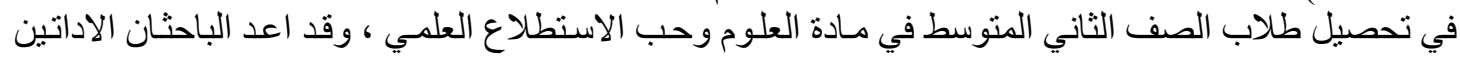
كالاتي : 1- الاختبار التحصيلي الإني

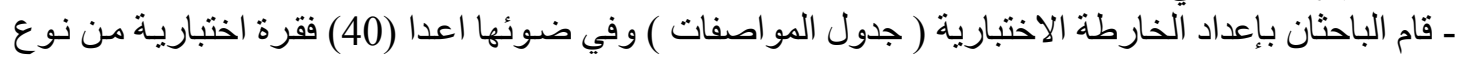

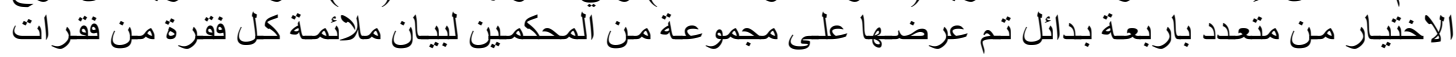




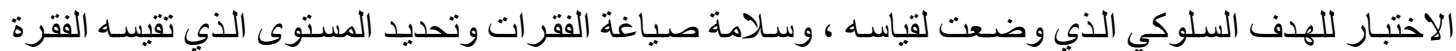

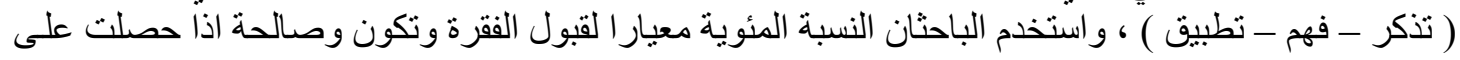

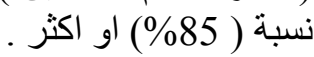
ـ تم تطبيق الاختبار على عبنة استطلاعبة مكونة من (100) طالباً من طلاب الصف الثان الثاني المنوسط في منوسطة

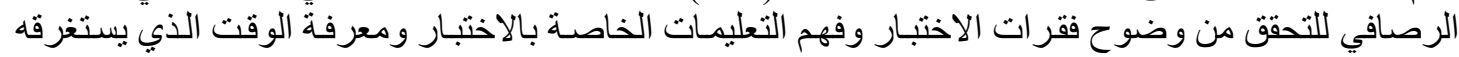

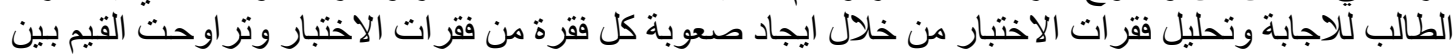

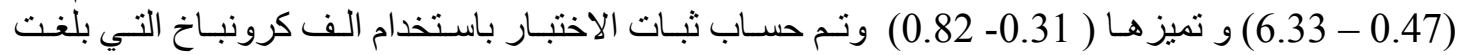
(0.785) ، وبهذا اكتسب الاختبار الصدق و الثين الثبات.

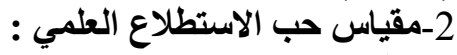
اعد الباحثنان مقياس حب الاسنطلاع الاسنطلّاع العلمي و على النحو الآتي :

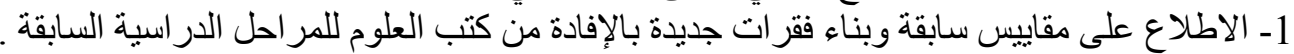

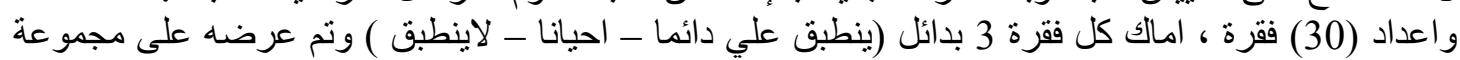

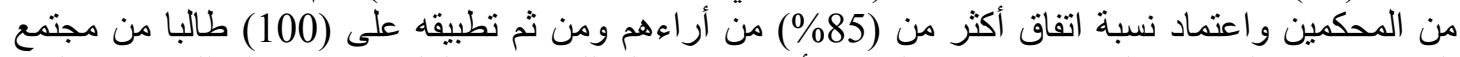

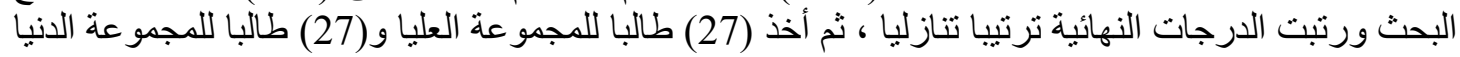

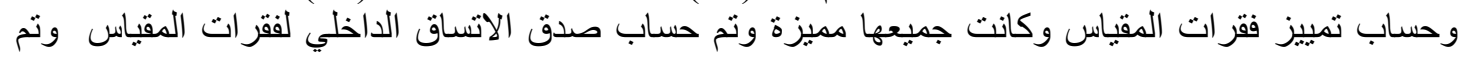
حساب ثبات المقياس باستخدام الفا كرونباخ وكانت القيمة (0.81) .

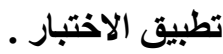

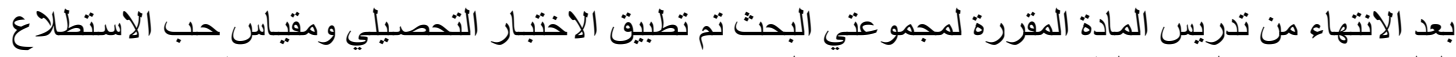

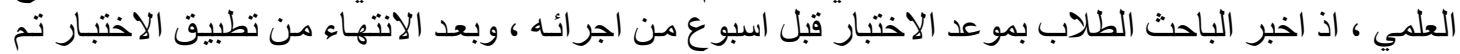
تحليل نتائجه لمعر ففة المردودات النهائية للتجربة.

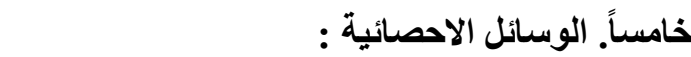

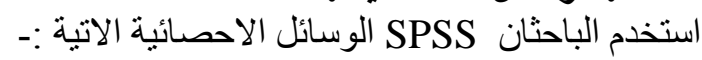
(t-test) -

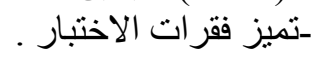
- صعوبة الفقر ات الات

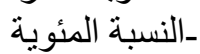
معامل الفا كرونباخ المتوسط الحسابي الانحر اف المعياري

\section{الفصل الرابع}

عرض النتائج وتفسبر العه

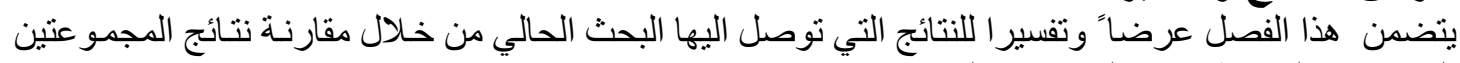

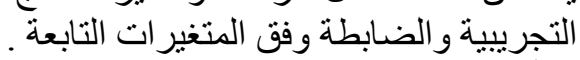

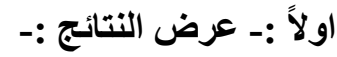

1. التحصيل - تم حساب t-test لمعرفة دلالة الفرق بين منوسطي درجات المجمو عتين ، كما في جدول (1).

\begin{tabular}{|c|c|c|c|c|c|c|c|}
\hline الدلالة عند 0.05 & p-value & $\mathrm{t}$ & $\mathrm{df}$ & Std. Deviation & Mean & $\mathrm{N}$ & المجمو عة \\
\hline \multirow[t]{2}{*}{ دالة } & \multirow{2}{*}{0.415} & \multirow{2}{*}{3.992} & \multirow[t]{2}{*}{84} & 4.91673 & 27.8571 & 42 & التجرييية \\
\hline & & & & 4.40899 & 23.8409 & 44 & الضابطة \\
\hline
\end{tabular}




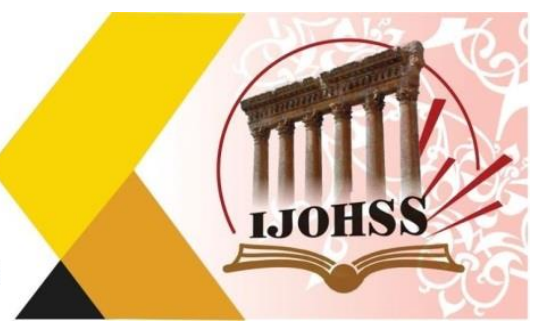

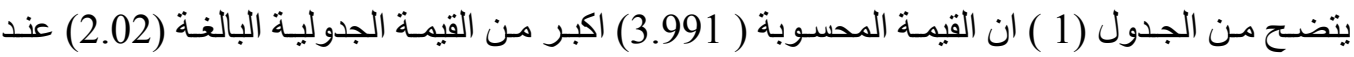

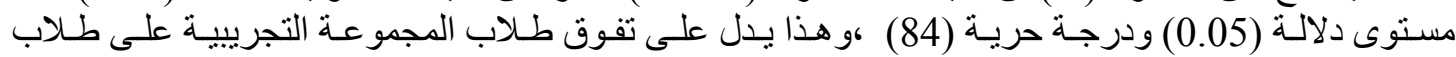
المجموعة الضابطة وبذللك ترفض الفرضية الصفرية الاولى.

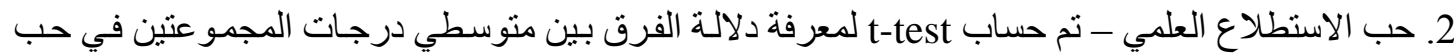
الاستطلاع العلمي ، كما في جدول (2) الاسئ (2). جدول (2) الدلالة الاحصائية لفرق منوسطي المجمو عتين في حب الاستطلاع العلمي.

\begin{tabular}{|c|c|c|c|c|c|c|c|}
\hline الدلالة عند 0.05 & p-value & $\mathrm{t}$ & df & Std. Deviation & Mean & $\mathrm{N}$ & المجمو عة \\
\hline \multirow[t]{2}{*}{ دالة } & \multirow{2}{*}{0.0000001} & \multirow{2}{*}{5.732} & \multirow[t]{2}{*}{84} & 1.66835 & 23.2619 & 42 & التجريبية \\
\hline & & & & 2.19876 & 20.8409 & 44 & الضابطة \\
\hline
\end{tabular}

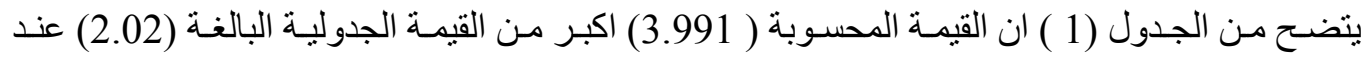

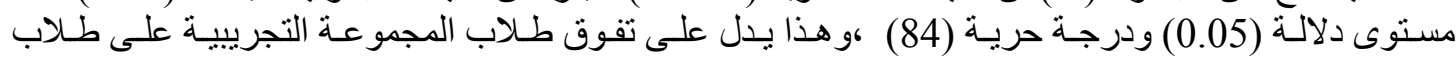
المجمو عة الضابطة وبذللك ترفض الفرضية الصفرية الثانية.

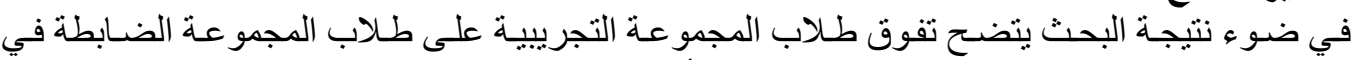
ثانياً :- تفسير النتائج :-

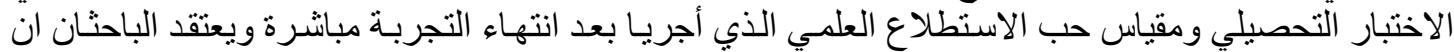

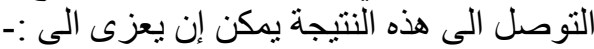

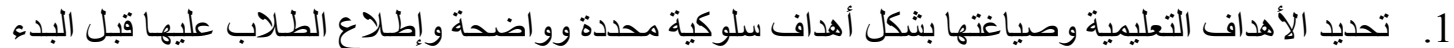

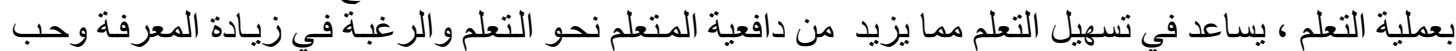

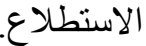

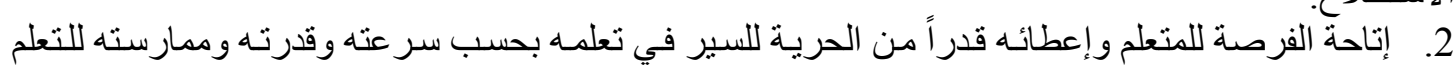

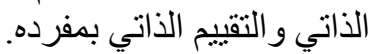
3. اضيفاء اجو اء من المنتعة والتفاعل اثناء التعامل مع الحقيبة التعليمية من خلال الحاسوب بجو هـادئ بعيد عن التوتر و الضغط . 4. تنظيم المادة العلمية وتقسيمها الى أجزاء صغيرة وفي خطوات متتابعة وبصيغة سهلة لها الأثر الأكبر في

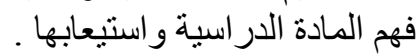

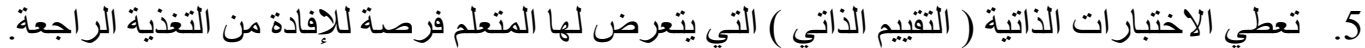

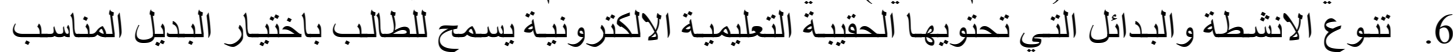

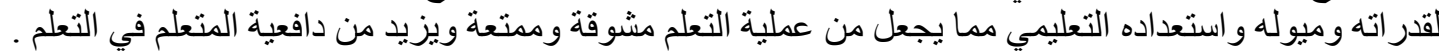

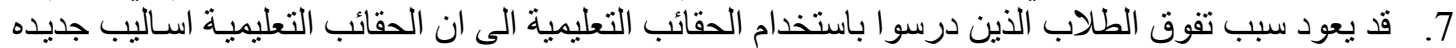

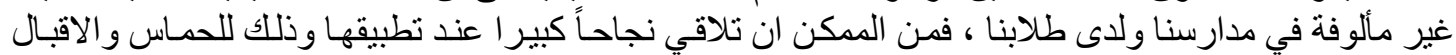

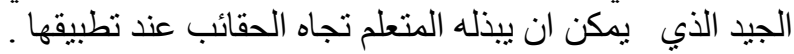

$$
\text { التوصيات }
$$

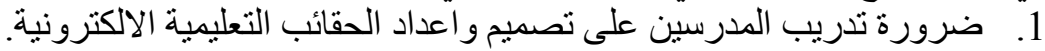

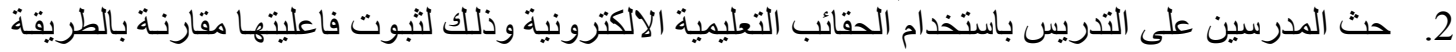
التقليدية

3. تزويد المدارس بالتقنيات التربوية الحديثة كالحاسوب و السبور ات الالكترونية لاستعمالها في التدريس. 4. تزويد المكتبات المدرسية والجامعية بالكتب و المر اجع التي تتضمن الاستر اتيجيات و طر ائق التدريس التي لتئي

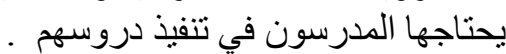




\section{لسبتمبر 2020}

Volume (15)
العدد (15)

September 2020

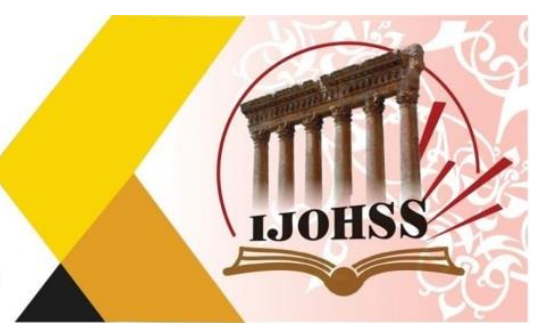

المقترحات المكيات

استكمالا للبحث الحالي يقترح الباحثنان اجر اء الاتي :-

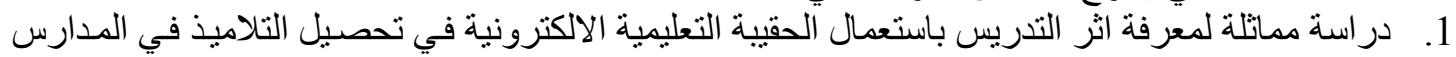
الابتدائية وطلبة المرحلة الاعدادية.

2. در اسة مقارنة بين الحقائب التعليمية الالكترونية وطر ائق التدريس الاخرى لغرض التعرف على فاعلية كل 3. منها . درات حول فاعلية الحقائب التعليمية الالكترونية في تدريس الطلبة الذين بعانون من صسعوبات الحضور الى المدارس.

$$
\begin{aligned}
& \text { المصادر 1. } \\
& \text { 1. رضوان ، شناكر فرج (2012) ـ علم النفس المدرسي ، مكتبة بهاء ، دمشق. }
\end{aligned}
$$

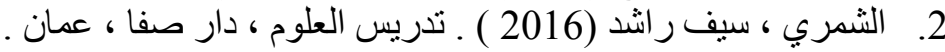

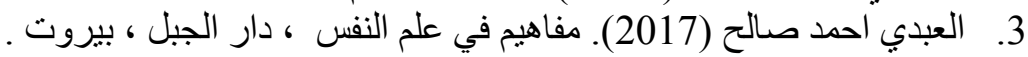

4. Moris , R.(2014).Learning system design . Mc Grow -Hill Book co . N.Y.

5. Novel B.(2009). Aspects of scientific Curiosityin in Secondary School students ' Journal of Science Education, Vol 65,no.2 .

6. Ramos , D. (2005). An Introduction in psychology, 2nd-ed.9 london,prentice-hall. 7. -Simo ,T. M.(2011).Methods of Teaching , New Jerseyc , prentice - hall , Englewood cliffs .

8. -Wolker K.(2016). Effect of level of Guriosity and age on The Exploratory Behavio of young children" Diss.Abs.Int vol .45 no.9 march. 